\title{
Vortex telegraph noise in high magnetic fields
}

\author{
E. Shung, T. F. Rosenbaum, and S. N. Coppersmith \\ The James Franck Institute and Department of Physics, The University of Chicago, Chicago, Illinois 60637 \\ G. W. Crabtree and W. Kwok \\ Materials Science Division, Argonne National Laboratory, Argonne, Illinois 60439
}

(Received 22 August 1997)

\begin{abstract}
We cool untwinned single crystals of $\mathrm{YBa}_{2} \mathrm{Cu}_{3} \mathrm{O}_{7-\delta}$ with columnar defects down to liquid-He temperatures and study the development of pinning in the strongly interacting Bose glass with local Hall-probe magnetometry. We are able to resolve discrete fluctuations in the local vortex density resulting from reconfigurations of the vortex assembly between metastable states nearby in energy. By varying the applied magnetic field, and therefore the mean vortex density, we gain microscopic information about vortex-vortex interactions. [S0163-1829(97)52142-4]
\end{abstract}

The effectiveness of columnar defects in enhancing the critical current density and the size of the irreversibility region in cuprate superconductors raises important questions about the nature of vortex-defect and vortex-vortex interactions in the presence of correlated disorder. The theoretical framework for this "Bose glass"' was worked out by Nelson and Vinokur, ${ }^{1}$ who mapped the system of vortices with columnar pins to the model system of quantum mechanical bosons in a time-independent two-dimensional (2D) potential. Three low-temperature phases are predicted as a function of the magnetic field. For internal fields below $B_{\phi}$, the field at which the number of vortices matches the number of defects, vortices localize on the disordered assembly of columnar pins. At $B_{\phi}$, the model predicts an incompressible "'Mott insulator', phase, characterized by an energy gap for adding the next vortex. ${ }^{1,2}$ Above $B_{\phi}$, interactions are important in determining pinning, and may result in a second Bose glass state in which interstitial vortices are weakly pinned by vortex-vortex interactions. ${ }^{3}$ Clear experimental signatures of each field regime occur in the magnetization relaxation, ${ }^{4,5}$ but microscopic information about the nature of pinning has not been accessible. This is particularly true for $B>B_{\phi}$, where strongly localized vortices at defects and weakly confined interstitial vortices intricately combine to carve out the generalized pinning potential.

When frozen into the Bose glass, the vortex assembly settles into some metastable local minimum in the free energy. Further relaxation to metastable states with lower free energy occurs on a time scale longer than the cooling time. Vortex fluctuations occur on much shorter time scales and have two components: broadband phononlike fluctuations about the local free-energy minima, and infrequent but fast fluctuators resulting from hops between local free-energy minima separated by an activation barrier. Both components are intrinsic features of pinning and are strongly influenced by vortex-vortex interactions.

Fluctuations in the local vortex density are detectable by local magnetic-field probes. Using a high sensitivity superconducting quantum interface device (SQUID) loop magnetometer, Clarke and co-workers ${ }^{6}$ have measured fluctuations due to thermally generated vortices in $\mathrm{YBa}_{2} \mathrm{Cu}_{3} \mathrm{O}_{7-\delta}$ (with- out columnar defects) in zero field just below the superconducting transition temperature. They resolve both the spectral content of the broadband noise and two-state fluctuators with frequencies up to $1 \mathrm{kHz}$. SQUID magnetometers, however, are limited to low-field applications.

Although significantly less sensitive, miniature Hall probes can access the high-field regime where vortex-vortex interactions become significant. Longer integration times and lower measurement frequencies $(<1 \mathrm{~Hz})$ are required to obtain acceptable flux resolution. These constraints make a spectral analysis of the broadband noise uninformative, but low-frequency fluctuators are resolvable, providing a means to explore the details of pinning in the strong interaction regime. Even on the micron length scale, the gaussmeters average over the motion of tens of thousands of vortices. Hence, to prevent a proliferation of states indistinguishable by the Hall probe, liquid-He temperatures are necessary to immobilize the majority of vortices and drastically reduce the space of accessible states.

In this paper we describe a systematic investigation of vortex fluctuators at high $H$ and low $T$. We find several trends with increasing field that indicate a strong role played by interactions in determining the pinning environment: fluctuator events increase in frequency and size, an entry-exit rate asymmetry develops, and both the entropy and the energy scales increase. Moreover, the temperature dependence of the event rate is nonmonotonic, a result hard to reconcile with a single vortex picture.

Columnar defects along the $c$ axis were introduced into an untwinned single crystal of $\mathrm{YBa}_{2} \mathrm{Cu}_{3} \mathrm{O}_{7-\delta}$ by irradiation with $700 \mathrm{MeV}$ Sn ions. The defects extend from face to face of the regularly shaped rectangular platelet, which has dimensions of $250 \mu \mathrm{m} \times 470 \mu \mathrm{m}$ in the $a-b$ plane and thickness $20 \mu \mathrm{m}$ along the $c$ axis. The irradiation dosage was chosen to produce a mean defect density $B_{\phi}=2 \mathrm{~T}$. We measured fluctuations in the local vortex density over a range of temperatures, $0.3 \mathrm{~K}<T<1.5 \mathrm{~K}$, and for a series of applied magnetic fields up to $8 \mathrm{~T}$, using a granular thin-film bismuth Hall probe. ${ }^{7}$ The sample was affixed to a $3 \mu \mathrm{m} \times 3 \mu \mathrm{m}$ active area gaussmeter with a thin layer $(<1 \mu \mathrm{m})$ of vacuum grease and carefully positioned so that the probe was at a point of 


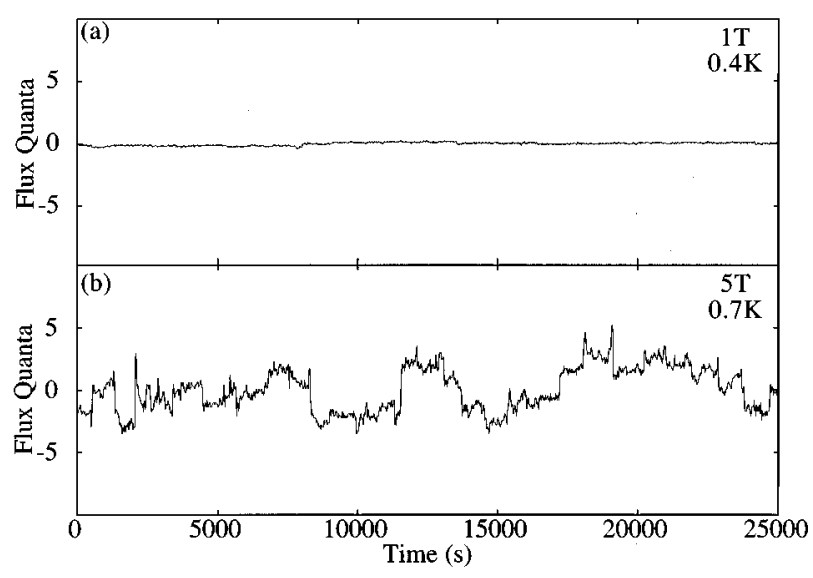

FIG. 1. Change in flux quanta under the micro-Hall probe vs time for (a) applied field $H=1 \mathrm{~T}$ and temperature $T=0.4 \mathrm{~K}$, and (b) $H=5 \mathrm{~T}$ and $T=0.7 \mathrm{~K}$. At low field, flux motion is by tunneling on small length scales and telegraph noise is not detectable.

symmetry, equidistant from either short edge, and halfway from the center of the crystal to one long edge. Only the differential Hall coefficient of the gaussmeter at each applied field needed to be calibrated in order to convert the measured Hall resistance to local vortex density. Using an ac resistance bridge with a $10 \mathrm{~s}$ time constant, we were able to obtain nearly single vortex resolution.

The near equilibrium Bose glass state was prepared by cooling the crystal in an applied magnetic field through $T_{c}$ to liquid-He temperatures over a $24 \mathrm{~h}$ period. After stabilizing at the target temperature, the local vortex density was monitored for up to $10^{5} \mathrm{~s}$ and sampled at $10 \mathrm{~s}$ intervals to generate a single time series. For each field preparation, time series were acquired for the desired range of temperatures. The procedure was then repeated for the next field.

We plot in Fig. 1 representative time series for $H=1$ and $5 \mathrm{~T}$, two fields for which the magnetization relaxation rates are nominally the same, lying on either side of the relaxation minimum at $B_{\phi}$. The $1 \mathrm{~T}$ time series, which is representative of those taken for $B<B_{\phi}$, exhibits no remarkable features, consisting entirely of broadband Gaussian noise at levels consistent with systematic instrumental noise. The absence of events below $B_{\phi}$ is in accord with the view ${ }^{4}$ that when all vortices are pinned on columnar defects, then flux motion is by quantum tunneling, which is confined to short distances by the length scale dependence of the Euclidean action. In sharp contrast, for $B>B_{\phi}$ the time series includes infrequent, but instantaneous (within our temporal resolution) and discrete changes in flux quanta, reminiscent of telegraph noise from electrons in Si metal-oxide-semiconductor-field-effect transistors (MOSFET's). ${ }^{8}$ These discrete fluctuator events correspond to those reconfigurations of the vortex assembly in the vicinity of the active area that produce resolvable changes in the local vortex density. Since we observe only the net change in vortex number, we cannot distinguish between events involving a single vortex hopping near the edge of the active area or a cascade of vortices entering one side of our probe and leaving by the other. Regardless, the observation of resolved fluctuator events implies the net motion of vortices over a length scale exceeding the penetration depth $(>0.1 \mu \mathrm{m})$.

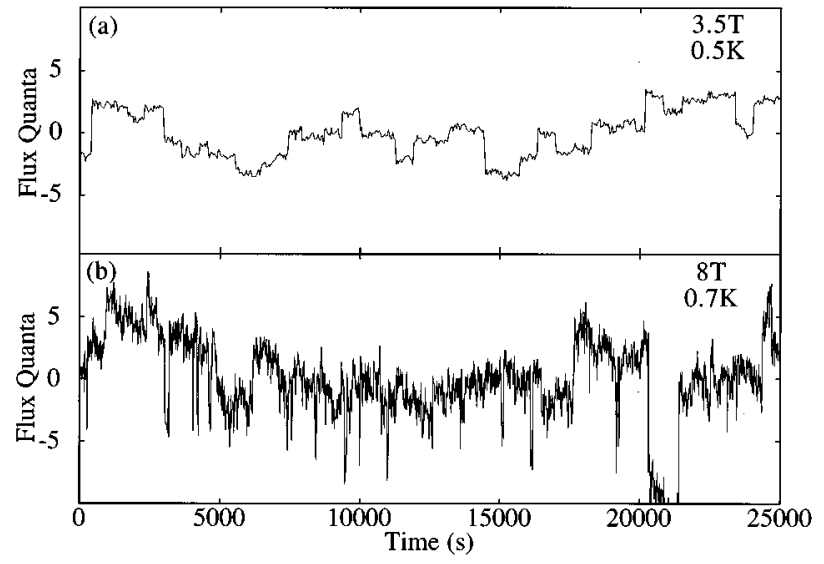

FIG. 2. Change in flux quanta vs time for (a) applied field $H$ $=3.5 \mathrm{~T}$ and temperature $T=0.5 \mathrm{~K}$, and (b) $H=8 \mathrm{~T}$ and $T=0.7 \mathrm{~K}$. The event sizes, rates, and up-down asymmetry all increase with increasing $H$.

The events above $B_{\phi}$ presumably correspond to thermal activation of interstitial vortices. In this strong interaction regime, fluctuators depend sensitively on magnetic field. We plot in Fig. 2 representative time series for $H=3.5$ and $8 \mathrm{~T}$. Several features develop with increasing applied field. Fluctuator events are larger and more frequent, and they exhibit an entry-exit rate asymmetry at $8 \mathrm{~T}$. A general increase in broadband noise is external and attributable to the decreasing sensitivity of the Hall probe at higher fields.

In order to quantify these trends, we identified for each time series every fluctuator event and tabulated its size and its time of occurrence, resulting in a histogram of change in vortex number between two consecutive measurements. A large central peak due to broadband noise was fit to a Gaussian, and a cut was made at the point where the fit curve yielded one or more events. Due to the increased systematic noise, the cut size was larger at higher fields. Excess counts passing this cut were identified as events.

The distribution of event sizes is symmetric between entry and exit events at all fields. The mean size of the events increases with field, from $2.2 \pm 0.6$ vortices at $3.5 \mathrm{~T}$ to 6.3 \pm 1.4 vortices at $8 \mathrm{~T}$. This increase in event size is expected. As the vortex density is increased, vortex-vortex interactions grow stronger, making it increasingly difficult for individual vortices to act independently, and favoring collective vortex motion. ${ }^{1}$ Systematic noise broadens the distribution of event sizes, preventing us from specifying whether events are truly in terms of flux quanta.

If a time series resulted from a true two-state fluctuator, then at each moment, based upon the value of the observable, the system could be assigned to either the "up" state or the "down" state. At first glance, our time series defy any such simple identification of states. In a more general sense, however, these time series do mimic a two-state system: with very high probability, entry events (down to up transitions) are followed by exit events (up to down transitions). With this looser identification of states, we have a framework for interpreting the data.

Beginning with transition rates, we define the entry (exit) rate as the inverse of the average time spent in the down (up) state before a transition to the up (down) state. In general, 


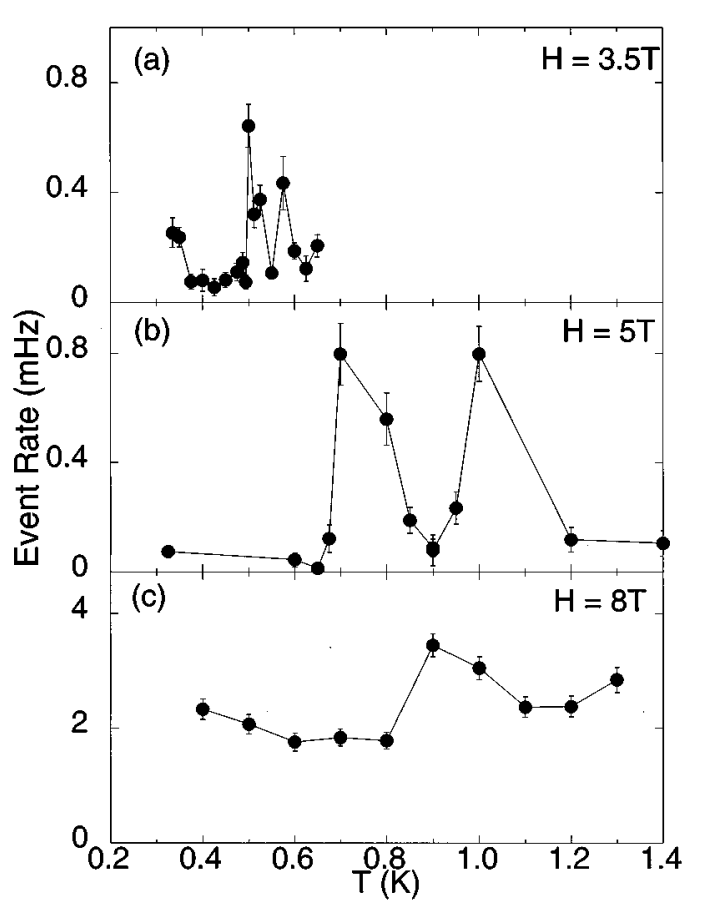

FIG. 3. Event rate vs temperature $T$ for (a) $H=3.5 \mathrm{~T}$, (b) $H$ $=5 \mathrm{~T}$, and (c) $H=8 \mathrm{~T}$. The non-Arrhenius form signals the collective nature of the fluctuations.

these rates need not be the same. We see a large asymmetry develop at $8 \mathrm{~T}$ in the entry-exit rate and, within uncertainty, none at lower fields. The large number of interstitial vortices cannot be deformed as easily and they develop a restoring force to the movement of flux which acts to increase the local density. The actual direction of the asymmetry is not significant, since a higher entry rate under the active area likely means a higher exit rate nearby. More significant is the tendency of the vortex assembly to develop this elastic response, which is again indicative of collective motion.

Despite the changes in event size, rate, and asymmetry with increasing $H$, the temperature dependence of the event rate has the same qualitative form at all $H$. We make this comparison for $H=3.5,5$, and $8 \mathrm{~T}$ in Fig. 3, where all the data are shown over the same range of temperature. The event rate is taken simply as the total number of events in a given time series divided by the duration of the time series. At each $H$, the event rate rises steeply with increasing $T$, with an onset temperature which increases with increasing field. The frequency of events also increases with increasing $H$; note that the baseline at $H=8 \mathrm{~T}$ is more than an order of magnitude larger than the baselines at $H=3.5$ and $5 \mathrm{~T}$. Moreover, a double-peaked structure is clearly defined at the lower fields and appears probable at $8 \mathrm{~T}$ if the temperature range could be extended.

We have made checks to ensure the robustness of these results. We have repeated the measurements for select temperatures at each field (a complete set of data for each $H$ represents many months of data acquisition time), verifying that the time series were qualitatively repeatable. Despite the reconfiguration expected from recurrent field cooling, it appears that the presence of strong quenched disorder in the form of columnar defects provides a common template for each field cooled preparation. The reproducibility of the local

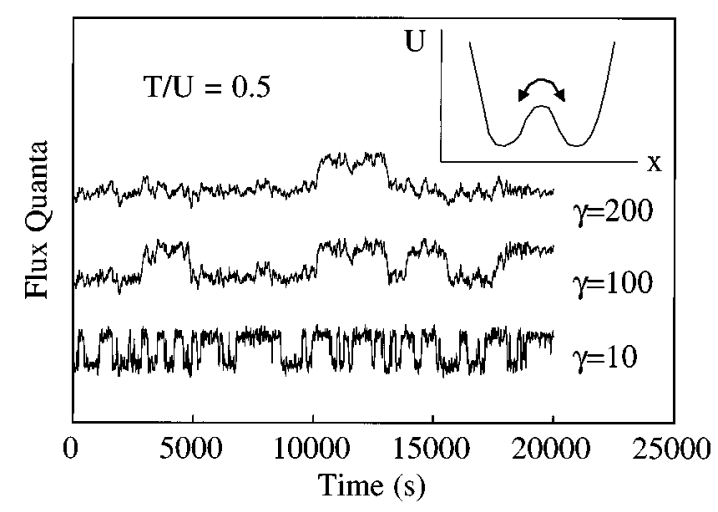

FIG. 4. Results of a Langevin equation simulation [Eq. (1) in the text] for a series of viscosities $\gamma$ at fixed temperature. Inset: model double-well potential.

free-energy landscape also has been inferred in studies of superconducting films, ${ }^{9}$ and it may help explain the common two-peak structure in the temperature dependence at each field.

The data of Fig. 3 are difficult to understand in terms of simple thermal activation of vortices in a generalized twostate potential. For an activated two-state fluctuator, the Arrhenius rate $\Gamma=\omega_{0} \exp \left\{-U / k_{B} T\right\}$, where $\omega_{0}$ is the attempt frequency and $U$ is the activation barrier. With $\omega_{0}$ fixed, a higher activation barrier at higher field shifts the onset temperature higher, but decreases the rate. It is more appropriate to replace the activation barrier by the free-energy barrier $F=U-T S$. Then, the measured higher event rate can be reconciled with the measured higher onset temperature. The entropy $S$ increases with increasing $H$ as the number of possible configurations grows with vortex density, permitting $\Gamma$ to grow as $\exp \left\{S / k_{B}\right\}$.

More problematically, the Arrhenius formula predicts an event rate which increases monotonically with increasing $T$. In practice, there is an instrumental cutoff which can result in a nonphysical peak: as the event rate approaches the sampling rate, telegraph events are no longer resolvable in time and a sharp drop in the observed rate can ensue. This explanation is not germane here, however, as the peak rate observed in Fig. 3 varies from 0.8 to $4 \mathrm{mHz}$, a factor of 25 to 100 below the sampling rate of $100 \mathrm{mHz}$.

One possible mechanism for this markedly non-Arrhenius behavior is thermally induced changes in the actual composition of the (self-organized) two-level states, posited to cause an observed monotonically decreasing fluctuation rate with increasing $T$ in $\mathrm{NbN}-\mathrm{MgO}-\mathrm{NbN}$ tunnel junctions with a high density of active defects. ${ }^{10}$ Alternatively, a crossover between rare jumps over a high barrier to diffusional dynamics may account for the nonmonotonic characteristic of Fig. 3. A simple double-well model for a two-state fluctuator system (Fig. 4 inset), governed by the Langevin equation

$$
\gamma(d x / d t)=-d U / d x+\eta(t),
$$

offers some insight. Here, $x$ is a generalized coordinate representing the vortex density, $U$ is the generalized potential, $\gamma$ is the friction coefficient, and $\eta(t)$ is a noise term satisfying $\langle\eta(t)\rangle=0,\left\langle\eta(t) \eta\left(t^{\prime}\right)\right\rangle=\gamma k_{B} T \delta\left(t-t^{\prime}\right)$, where \langle\rangle denotes a time average. An event occurs when a rare large fluctuation 
launches the system just beyond the barrier and the system slides down to the other well. Telegraph noise appears if this process is faster than the measurement time scale. In the limit of large $\gamma$ and/or large $T$, however, the system undergoes diffusive motion and no sudden events are observed. In principle, a peaked event rate vs temperature can result from this crossover.

We show in Fig. 4 the results of a computer simulation of Eq. (1) for $T / U_{0}=0.5$ and $\gamma=10,100$, and 200 in units of ( $U_{0} t / x_{0}^{2}$ ), where $U_{0}$ is the barrier height, $x_{0}$ is the distance between the wells, and $t$ is the sampling time. Well-defined telegraph noise evolves into long times of quiet punctuated by rare events, a progression from an event-rate peak to a nonzero trough (cf. Fig. 3). Agreement between this model and the experiment requires a strongly temperaturedependent effective viscosity, which could result from collective effects.

The simulation results demonstrate that collective vortex behavior can account for a peak in the event rate under appropriate conditions. Given the large number of vortices under the gaussmeter, the observed double-peak structure might then result from two distinct two-state fluctuators with activation barriers close by in energy. More fundamentally, two coupled peaks in the event rate may follow from the fact that vortices in $\mathrm{YBa}_{2} \mathrm{Cu}_{3} \mathrm{O}_{7-\delta}$ are lines, not particles, leading to a quantum-mechanical splitting of the generalized two-level potential via the Nelson-Vinokur mapping. ${ }^{1,11}$

In summary, discrete vortex fluctuations in high- $T_{c}$ superconductors can be imaged at the level of the fundamental unit of flux by Hall microprobes in the high-field regime inaccessible to SQUID magnetometry. Several trends emerge with increasing magnetic field: fluctuator events increase in frequency and size, an entry-exit rate asymmetry develops, and both the entropy and the energy scales increase. The non-Arrhenius temperature dependence of the event rate emphasizes the importance of collective effects at large vortex density. This technique should open a new window into the microscopics of pinning and vortex-vortex interactions in the Bose glass.

We are grateful to A. Abrikosov, H. Jaeger, A. Koshelev, E. Nowak, M. Tarlie, and V. Vinokur for illuminating discussions. This work was supported by the National Science Foundation (Grant No. DMR91-20000) through the Science and Technology Center for Superconductivity. G.W.C. and W.K. acknowledge support from the U.S. Department of Energy, Basic Energy Sciences-Materials Science under Contract No. W-31-109-ENG-38.
${ }^{1}$ D. R. Nelson and V. M. Vinokur, Phys. Rev. Lett. 68, 2398 (1992); Phys. Rev. B 48, 13060 (1993).

${ }^{2}$ U. Täuber, H. Dai, D. R. Nelson, and C. M. Leiber, Phys. Rev. Lett. 74, 5132 (1995). A recent preprint (C. Wengel and U. Täuber) indicates that only a soft Coulomb gap may survive in the limit of penetration depth $>$ vortex spacing.

${ }^{3}$ L. Radzihovsky, Phys. Rev. Lett. 74, 4923 (1995); 74, 4919 (1995).

${ }^{4}$ K. M. Beauchamp et al., Phys. Rev. Lett. 75, 3942 (1995); Phys. Rev. B 52, 13025 (1995).
${ }^{5}$ E. R. Nowak et al., Phys. Rev. B 54, R12 725 (1996).

${ }^{6}$ M. Johnson et al., Phys. Rev. B 42, 10792 (1990); M. J. Ferrari et al., J. Low Temp. Phys. 94, 15 (1994).

${ }^{7}$ G. T. Seidler et al., Phys. Rev. Lett. 70, 2814 (1993).

${ }^{8}$ R. E. Howard et al., IEEE Trans. Electron Devices ED-32, 1669 (1985).

${ }^{9}$ E. R. Nowak, N. E. Israeloff, and A. M. Goldman, Phys. Rev. B 49, 10047 (1994).

${ }^{10}$ L. Pesenson et al., Phys. Rev. Lett. 67, 2866 (1991).

${ }^{11} \mathrm{~V}$. Vinokur (private communication). 